\title{
Alpelisib in the Treatment of Breast Cancer: A Short Review on the Emerging Clinical Data
}

This article was published in the following Dove Press journal:

Breast Cancer: Targets and Therapy

\section{Avan J Armaghani \\ Hyo Sook Han}

Department of Breast Oncology, Moffitt McKinley Outpatient Center, H. Lee Moffitt Cancer Center and Research Institute, Tampa, FL 336/2, USA
Correspondence: Avan J Armaghani Email avan.armaghani@moffitt.org

\begin{abstract}
Hormone receptor positive, human epidermal growth factor receptor 2 negative (HR+/HER2 negative) breast cancer accounts for over $70 \%$ of all breast cancers. There has been much advancement in the treatment of HR+/HER2 negative metastatic breast cancer (MBC), in particular the development of more tailored and targeted therapies. Recently, greater understanding of the role of phosphatidylinositol 3-kinase (PI3K)/AKT/mammalian target of rapamycin (mTOR) pathway in breast cancer has led to the development of PI3K inhibitors, which have proven to be effective in the treatment of HR+/HER2 negative MBC. In this review, we will discuss the role of the PI3K/AKT/mTOR pathway in breast cancer and therapies that have been developed to inhibit PI3K. We will discuss in detail the development of PI3K inhibitor alpelisib, indications for use in HR+/HER2 negative MBC, safety and tolerability and the future direction of this therapy in the treatment of breast cancer.
\end{abstract}

Keywords: PI3K, PIK3CA, alpelisib

\section{Introduction}

Breast cancer is the leading cause of cancer and the second leading cause of cancer death in women. ${ }^{1}$ Hormone receptor positive, human epidermal growth factor receptor 2 negative (HR+/HER2 negative) breast cancer accounts for over $70 \%$ of all breast cancers. ${ }^{1}$ Recently, there has been a paradigm shift in the treatment of HR +/HER2 negative metastatic breast cancer (MBC) with development of targeted therapies, which have led to patients living longer and with less toxicity. Cyclin dependent kinase 4/6 inhibitors (CDK 4/6i) including palbociclib, ribociclib and abemaciclib, in combination with either aromatase inhibitor (AI) or fulvestrant, have revolutionized the treatment of $\mathrm{HR}+/ \mathrm{HER} 2$ negative $\mathrm{MBC}$ and has become the standard first-line treatment in this setting. ${ }^{2-5}$ Additional treatment options in HR +/HER2 negative MBC include estrogen receptor (ER) antagonist fulvestrant as a single agent and mammalian target of rapamycin (mTOR) inhibitor everolimus, both of which have shown to be an effective treatment in HR+/HER2 negative MBC. ${ }^{6,7}$ However, resistance to endocrine and targeted therapies ultimately occurs, warranting the need for new therapeutic strategies. In this review, we will discuss the role of the Phosphatidylinositol 3-kinase (PI3K)/AKT/mammalian target of rapamycin (mTOR) pathway in breast cancer and the development of PI3K inhibitors as a therapeutic option for breast cancer patients. We will also discuss in detail Alpelisib, the first approved PI3K inhibitor for the treatment of HR+/HER2 negative $\mathrm{MBC}$. 


\section{The PI3K/AKT/mTOR Pathway and Breast Cancer}

The PI3K/AKT/mTOR pathway plays a critical role in regulating cell proliferation, growth and survival. In breast cancer, therapeutic strategies have been developed to target these three important sites in this pathway. BOLERO-2 was a randomized, double-blinded Phase III placebo controlled study that compared the mTOR inhibitor everolimus in combination with exemestane versus exemestane and placebo in HR+/HER2 negative MBC. ${ }^{7}$ Median progression free survival (PFS) by central assessment was improved from 4.1 month to 10.6 months (HR 0.36, p<0.001). ${ }^{7}$ The AKT inhibitor capivasertib was studied in the Phase II FAKTION trial. ${ }^{8}$ This study compared capivasertib plus fulvestrant versus fulvestrant and placebo in HR+/HER2 negative MBC. Median PFS was 10.3 months in the capivasertib group versus 4.8 months in the placebo group (HR 0.58, $\mathrm{p}=0.0018) .{ }^{8}$

PI3Ks are a family of lipid kinases. ${ }^{9}$ Stimulation of receptor tyrosine kinase activates this pathway, which in turn triggers activation of PI3K (Figure 1). ${ }^{10}$ Class I PI3K is further divided into subclass IA and IB. ${ }^{11}$ Class IA PI3Ks are heterodimers consisting of a $110 \alpha$ catalytic subunit and $\mathrm{p} 85$ regulatory subunit. ${ }^{11}$ There are four catalytic isoforms of PI3K including $\alpha, \beta, \gamma$ and $\Delta .{ }^{11}$
PIK3CA is an oncogene that encodes the p110 $\alpha$ catalytic isoform. ${ }^{11}$ Three common mutations in PIK3CA include E545K and E542K on exon 9 and H1047R on exon 20. ${ }^{11}$ Mutation of PIK3CA leads to gain of function activation of the $\mathrm{p} 110 \alpha$ catalytic isoform, which causes a downstream effect that ultimately leads to unregulated cell growth, proliferation and survival. ${ }^{11}$

PIK3CA has been found to be one of three somatic mutations that occur commonly in breast cancer. ${ }^{12}$ Approximately $20-50 \%$ of all breast cancers harbor a PIK3CA mutation. ${ }^{11}$ HR positive and HER2 positive breast cancers are the two subtypes that exhibit this mutation most commonly, with incidence $35 \%$ and $23 \%$, respectively. ${ }^{11,13}$ Greater understanding of the role of PIK3CA mutation in cancer cell growth and survival has led to the development of targeted therapeutics aimed at directly inhibiting the PI3K pathway.

\section{PI3K Inhibitors}

\section{Pan-PI3K Inhibitors}

Early strategies of inhibiting PI3K have involved paninhibition of all four PI3K isoforms. Pan-inhibition can potentially be effective in a broad range of tumor types, but it can also be associated with greater toxicity.

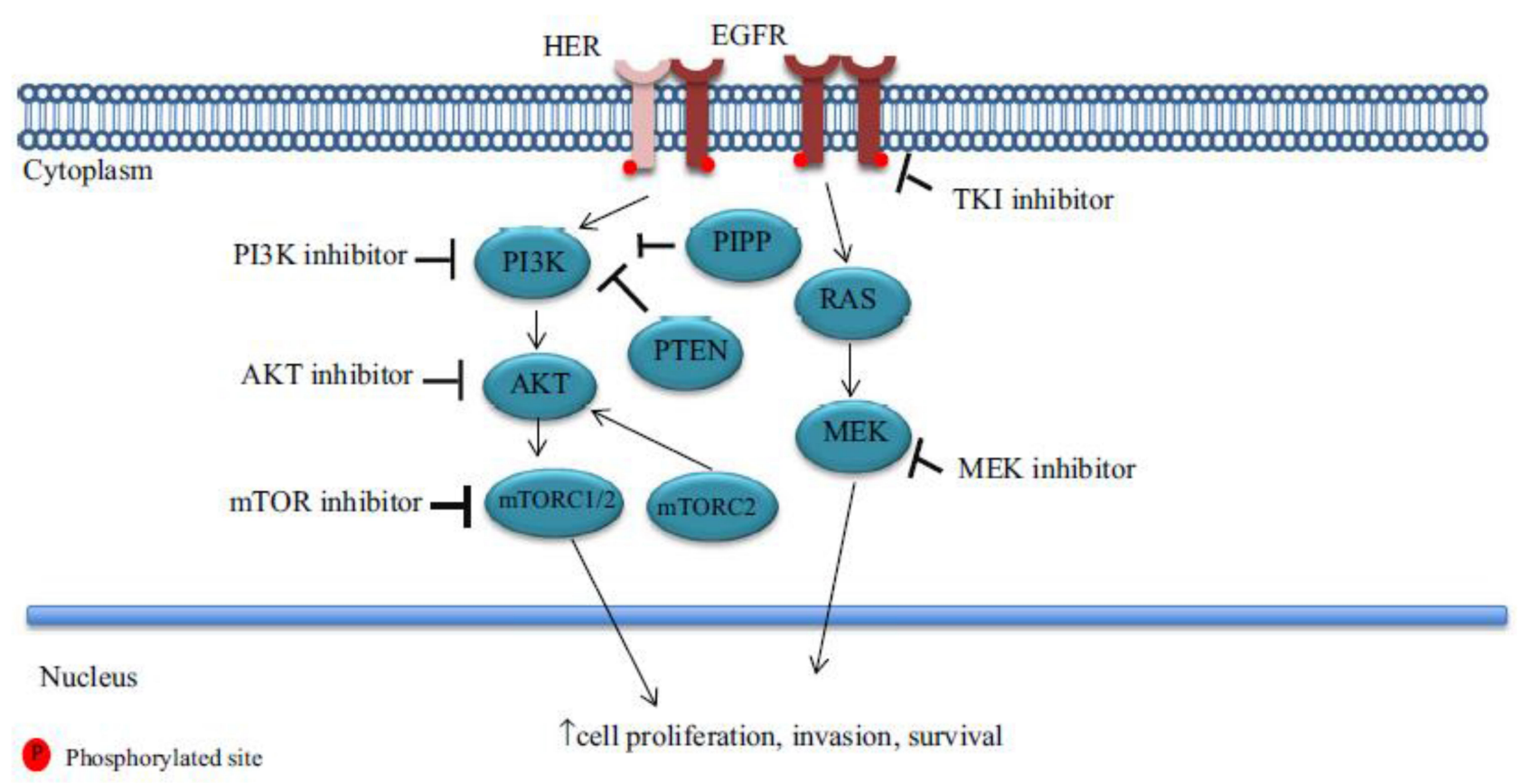

Figure I Therapeutic strategies have been developed to target various steps in the PI3K/AKT/mTOR pathway, thereby blocking cell proliferation, growth and survival. Note: Reprinted by permission from Springer Nature: Springer, Breast Cancer Res Treat, Costa, RLB, Han HS, and Gradishar WJ, Targeting the PI3K/AKT/mTOR pathway in triple negative breast cancer: a review. Copyright (20I8). 169(3): p. 397-406. Available from: https://link.springer.com/article/I0.1007/s I0549-0I8-4697-y. ${ }^{29}$ 


\section{Buparlisib}

Buparlisib is an oral pan PI3K inhibitor. ${ }^{14}$ The BELLE-2 study was a randomized, double-blind- placebo controlled Phase 3 study evaluating the efficacy of buparlisib in breast cancer. ${ }^{14}$ In this study, 1147 patients with HR+/HER2 negative inoperable locally advanced or MBC whose disease had progressed on or after AI and who had received up to one previous line of chemotherapy were included. Patients were randomly assigned to receive fulvestrant with or without buparlisib. The addition of buparlisib improved median PFS in the total patient population from 5.0 months to 6.9 months (HR 0.78, p=0.00021). In patients with PI3K activated pathway, median PFS was 6.8 months in the combination group compared with 4.0 months in fulvestrant alone group (HR 0.76, $\mathrm{p}=0.014$ ). The most common grade 3-4 adverse events (AEs) in the combination group were increased liver functions tests (LFTs), hyperglycemia and rash. $^{14}$

In the phase III BELLE-3 study, 432 post-menopausal women were randomly assigned to fulvestrant with or without the addition of buparlisib. Patients had HR+/HER2 negative locally advanced or MBC who had relapsed on or after endocrine therapy and mTOR inhibitor. The addition of buparlisib led to improvement in median PFS from 1.8 months to 3.9 months (HR 0.67, $\mathrm{p}=0.00030$ ). The most frequent grade 3-4 AEs in the buparlisib group included elevation in LFTs, hypertension and fatigue. ${ }^{15}$ Due to toxicities and safety issues with only modest PFS improvement, this drug did not move forward in further development.

\section{Pictilisib}

Pictilisib is also a pan PI3K inhibitor. The phase II PEGGY trial randomized 183 pre- and post-menopausal patients with $\mathrm{HR}+/ \mathrm{HER} 2$ negative MBC to receive chemotherapy (paclitaxel) with either pictilisib or placebo. There was no statistically significant improvement in PFS with the addition of pictilisib. In the intention to treat (ITT) population, median PFS was 7.8 months in the placebo group compared with 8.2 months with the addition of pictilisib (HR 0.95, $\mathrm{p}=0.83$ ). In patients with PIK3CA mutation, the median PFS was 5.8 months in the placebo group compared with 7.3 months with the addition of pictilisib (HR 1.06, $\mathrm{p}=0.88$ ). Pictilisb was associated with more frequent serious AEs and dose reductions/discontinuations compared to placebo. ${ }^{16}$

\section{Isoform-Specific PI3K Inhibitors}

Isoform-specific PI3K inhibitors may offer the opportunity for higher doses and potentially greater efficacy with less toxicity compared to pan PI3K inhibitor. ${ }^{11}$ Taselisib is a beta-sparing PI3K inhibitor that was studied in the phase III SANDPIPER clinical trial. ${ }^{17}$ In this study, 516 postmenopausal women were randomized to fulvestrant plus placebo or fulvestrant plus taselisib. The combination treatment group improved investigator assessed PFS from 5.4 months to 7.4 months (HR $0.70, p=0.0037$ ). The most common grade 3/4 AEs included diarrhea, hyperglycemia, colitis and stomatitis. ${ }^{17}$

Alpelisib (NVP-BYL719) is an oral bioavailable selective inhibitor of PI3K alpha. ${ }^{10}$ The safety and tolerability of this drug were first described in vivo in PIK3CA mutated solid tumors. ${ }^{10}$ This led to further studies evaluating the role of alpelisib in PIK3CA mutated breast cancer.

\section{Alpelisib in the Treatment of PIK3CA Mutated Breast Cancer}

Following preclinical data demonstrating tolerability and efficacy of alpelisib, the first in human phase Ia study was performed to show efficacy and tolerability of alpelisib in PIK3CA altered advanced solid tumors. ${ }^{18}$ In this study, 134 patients with unresectable, advanced solid tumors were enrolled. The most common solid tumor types were breast $(26.9 \%)$, colorectal $(26.1 \%)$ and head and neck $(14.2 \%)$. Results showed favorable safety profile up to $400 \mathrm{mg}$ daily dose and 150mg twice daily dose. Objective tumor response was observed at a dose of $270 \mathrm{mg}$ or higher once daily. Overall response rate was seen in 8 patients $(6.0 \%)$, with partial response seen in seven patients and one complete response. In patients with $\mathrm{HR}+/ \mathrm{HER} 2$ negative breast cancer, median PFS was 5.5 months. The most commons AEs included hyperglycemia (52\%), nausea (51\%), decreased appetite (42\%), diarrhea $(40 \%)$ and vomiting $(31 \%)$. In the dose escalation phase, dose limiting toxicities (DLTs) occurred in nine total patients. DLTs included hyperglycemia ( $\mathrm{n}=2$ at $450 \mathrm{mg}$ once daily dose and $n=4$ at 200mg twice daily dose), nausea ( $n=2$ at $450 \mathrm{mg}$ once daily dose) and combined hyperglycemia and hypophosphatemia ( $\mathrm{n}=1$ at $150 \mathrm{mg}$ twice daily dose). ${ }^{18}$

As stated previously, the incidence of PIK3CA mutation in $\mathrm{HR}+$ breast cancer is approximately $35 \% .^{11,13} \mathrm{PI} \mathrm{K}$ inhibition by alpelisib has been shown to cause an increase in estrogen receptor transcription in breast cancer cells. ${ }^{19}$ Therefore, one strategy that has been studied is combination of $\mathrm{PI} 3 \mathrm{~K}$ inhibitor with endocrine therapy in PIK3CA mutated $\mathrm{HR}+$ breast cancers in order to achieve greater sustained therapeutic response compared to endocrine therapy alone. ${ }^{20}$ In a phase Ib study, escalating once-daily doses 
of alpelisib (300mg, 350mg and $400 \mathrm{mg}$ ) in combination with fixed dose fulvestrant $(500 \mathrm{mg})$ was studied in 87 postmenopausal women with PIK3CA altered or PIK3CA wild type HR positive MBC. ${ }^{20}$ Patients had received a median of five prior lines of antineoplastic therapy. The maximum tolerated dose (MTD) was $400 \mathrm{mg}$ and the $300 \mathrm{mg}$ once daily dose was determined to be the recommended Phase 2 dose. The median PFS in PIK3CA altered group with alpelisib was 9.1 months (95\% CI, 6.6-14.6) versus 4.7 months (95\% CI, 1.9-5.6) in wild type group. Overall response rate in the PIK3CA altered group was $29 \%$ and no objective tumor response was observed in the wild type group. The most common grade 3-4 AEs with alpelisib $400 \mathrm{mg}$ once daily dosing included hyperglycemia (22\%) and maculopapular rash $(13 \%){ }^{20}$

With evidence of the efficacy and tolerability of alpelisib as monotherapy and in combination with endocrine therapy, a phase 3 study was performed to further confirm these findings. ${ }^{21}$ The SOLAR -1 study was a randomized phase 3 study that sought to evaluate the efficacy and safe of alpelisib plus fulvestrant in patients with PIK3CA mutated HR+/HER2 negative who had received prior endocrine therapy but no prior chemotherapy. ${ }^{21} \mathrm{~A}$ total of 572 patients were enrolled in the study, 341 patients had a PIK3CA mutation. At a median follow-up of 20 months, the median PFS in patients with PIK3CA mutation was 11.0 months in the alpelisib plus fulvestrant combination group compared with 5.7 months in the fulvestrant alone group (HR $0.65, \mathrm{p}<0.001$ ). Overall response rate in patients with PIK3CA mutation was $26.6 \%$ in the combination group compared to $12.8 \%$ in the fulvestrant alone group. The most frequent grade 3-4 AEs in the alpelisib plus fulvestrant cohort included hyperglycemia (36.6\%) and rash $(9.9 \%)$. Grade 3 diarrhea occurred in $6.7 \%$ of patients in the combination group. No grade 4 diarrhea was reported. The rate of discontinuation in the combination group was $25 \%$ compared to $4.2 \%$ in fulvestrant alone group. ${ }^{21}$ The results from the SOLAR-1 phase 3 study led to the FDA approval on May 24, 2019 of alpelisib in combination with fulvestrant for postmenopausal women and men with HR+/HER2 negative, PIK3CA-mutated advanced or metastatic breast cancer following progression on or after an endocrine-based regimen. ${ }^{19}$

\section{Mechanisms of Resistance and Mechanisms of Enhanced Response to Alpelisib}

There are multiples mechanisms of resistance to PI3K inhibitors. The effect of isoform specific PI3K inhibitors, like alpelisib, can be circumvented by activating other isoforms and thereby promoting downstream signaling of the PI3K/AKT/mTOR pathway. ${ }^{22}$ Additional mechanisms include activation of downstream effectors like AKT or mTOR. $^{22}$

Loss of PTEN has also been shown to be a cause of resistance to PI3K inhibitors. In one study, circulating tumor DNA (ctDNA) and tumor tissue was analyzed from patients with HR+/HER2 negative MBC who participated in a phase I/II trial of alepelisib in combination with $\mathrm{AI} .^{23}$ The authors sought to identify mechanisms of resistance that could explain why a subset of patients lacked clinical benefit to alpelisib and rapidly progressed following initiation of treatment. Clinical benefit was only observed in patients who harbored a PIK3CA mutation. CtDNA was then used to identify PTEN mutations in $25 \%$ of patients. Three of these patients had PTEN loss in pre-treatment samples and all of whom had rapid progression of disease after start of treatment with alpelisib and AI therapy. It was also discovered in tumor xenograft models that mutations in ESR1 led to rapid progression following start of alpelisib and AI therapy. Further supporting the idea that there is cross talk between PI3K and ER signaling and mutations in ESR1 can further promote resistant and contribute to progression of disease. ${ }^{23}$

While there are mechanisms of resistance to alpelisib, there are also mechanisms of enhanced response to this therapy. $10-15 \%$ of all PIK3CA mutant tumors across all cancer types contain multiple PIK3CA mutations, mainly double PIK3CA mutations. ${ }^{24}$ Double PIK3CA mutations are commonly found in HR+/HER2 negative breast cancers. Double mutations in cis have shown to increase downstream PI3K pathway signaling and cell/tumor proliferation compared to single mutations. An analysis was performed looking at the ctDNA from the SANDPIPER study to detect the presence of PIK3CA mutations. Patients with a single PIK3CA mutation receiving taselisib had an overall response rate of $18.1 \%$. But patients with multiple PIK3CA mutations receiving taselisib had overal response rate of $30.2 \% .{ }^{24}$

Taken together, the above data suggest that identification of other mutations (ie, ESR1, PTEN) in addition to PIK3CA and quantifying the number of unique PIK3CA mutations may be necessary in order to create tailored therapeutic strategies for patients with $\mathrm{HR}+/ \mathrm{HER} 2$ negative MBC. 


\section{PIK3CA Mutation Testing}

The FDA has approved the Therascreen ${ }^{\mathbb{B}}$ PIK3CA mutation assay for detection of PIK3CA mutation in patients. ${ }^{25}$ This diagnostic test was used in the SOLAR-1 study and it detects 11 PIK3CA mutations in tumor tissue specimens and/or in ctDNA. ${ }^{21}$ However, there is considerable heterogeneity of PIK3CA mutations in breast cancer and there could potentially be additional PIK3CA mutations that have important clinical implications and are not detected on the Therascreen ${ }^{\circledR}$ assay. In one study, the Therascreen ${ }^{\circledR}$ panel detected four common PIK3CA mutations (H1047R, E545K, E542K, H1047L) comprising 67\% of all PIK3CA mutations in the study dataset. ${ }^{25}$ Furthermore, the Therascreen ${ }^{\circledR}$ assay was shown to detect $80 \%$ of patients who have a known PIK3CA mutation. In patients known to harbor double PIK3CA mutation, the Therascreen ${ }^{\circledR}$ assay identified both PIK3CA mutations in only $5 \%$ of patients. ${ }^{25}$ This study points to limitations of the Therascreen ${ }^{\circledR}$ assay and potentially $20 \%$ of patients with a known PIK3CA mutation would go undetected. Furthermore, it is unclear the clinical importance and benefit of alpelisib therapy in patients with PIK3CA mutations that are not identified by the Therascreen ${ }^{\circledR}$ assay but may be detected on other nextgeneration sequencing assays.

\section{Administration/Safety and Toxicity}

The starting dose of alpelisib is $300 \mathrm{mg}$ once daily. There are two dose reductions available which are $250 \mathrm{mg}$ once daily and 200mg once daily. Fulvestrant is administered $500 \mathrm{mg}$ IM on days 1, 15 and 29 and one monthly thereafter. ${ }^{19}$

The most frequent grade 3-4 AEs observed in patients who received alpelisib included hyperglycemia, severe cutaneous reaction and diarrhea. Additional adverse reactions that are listed in the FDA prescribing information include severe hypersensitivity and pneumonitis. Specific monitoring guidelines have been established to address these side effects. All patients must have a fasting plasma glucose (FPG) and HbA1C prior to initiation with alpelisib. FPG is then monitored once every week for the first 2 week and then at least every 4 weeks. HbAlc is monitored every 3 months and as clinically indicated. Patients with a history of controlled type 2 diabetes were included in SOLAR-1 trial but patients with type 1 and uncontrolled type 2 diabetes were excluded. Patients who develop grade 1 or greater hyperglycemia are recommended to initiate or intensify anti-hyperglycemia treatment. ${ }^{19}$

Severe cutaneous reaction including Stevens-Johnson syndrome (SJS) and erythema multiforme (EM) were reported in $0.4 \%$ and $1.1 \%$ of patients in SOLAR-1 study, respectively. Specific guidelines are outlined for patients who may develop cutaneous reaction, including initiation of topical and/or oral corticosteroids and oral antihistamines and interruption or permanent discontinuation of alpelisib. In a subgroup of patients in the SOLAR-1 study, 86 patients received prophylaxis including antihistamines and the incidence of all grade rash was $27 \%$ versus $54 \%$ in the overall population. Similarly, the incidence of grade 3 rash was $12 \%$ in the group of patients receiving prophylactic antihistamine compared to $20 \%$ in overall population. Therefore, prophylactic antihistamine is a consideration in patients initiating alpelisib. ${ }^{19}$

Diarrhea of all grades occurred in $58 \%$ of patients in the SOLAR-1 study and $6.7 \%$ patients experienced grade 3 diarrhea. There were no episodes of grade 4 diarrhea. Recommendations for the management of diarrhea include initiating and/or intensifying antidiarrheal medications and interruption alpelisib therapy. ${ }^{19}$

Pneumonitis is a less common adverse event with it occurring in $1.8 \%$ of patients receiving alpelisib in SOLAR-1 study. Symptoms such as shortness of breath, cough, or interstitial infiltrates on radiologic exam raise concern for pneumonitis in patients on alpelisib. Permanent discontinuation of alpelisib is advised in any patient who develops pneumonitis. ${ }^{19}$

\section{Future Directions}

CDK 4/6i (palbociclib, ribocilib or abemaciclib) in combination with either AI or fulvestrant is the standard first-line treatment for HR+/HER2 negative MBC. ${ }^{2-5}$ Greater understanding of the PI3K/AKT/mTOR pathway has led to the development of therapies that specifically target this pathway. Table 1 highlights clinical trials currently underway evaluating additional therapeutic strategies to target this pathway.

In the SOLAR-1 study, only 5-6\% of patients across all groups received previous treatment with a CDK $4 / 6 i^{21}$ Alpelisib has been approved in combination with fulvestrant in patient patients who have progressed on endocrine therapy. ${ }^{19}$ This raises the question about the role of alpelisib in patients who have progressed on CDK 4/6i. Clinical trials are currently ongoing to answer this question. Initial results from the BYLieve study were recently reported. ${ }^{26}$ This phase II open-label non-comparative 
Table I Ongoing Clinical Trials Investigating Therapies Targeting the PI3K/AKT/mTOR Pathway

\begin{tabular}{|c|c|c|c|c|c|c|c|c|}
\hline Therapy & $\begin{array}{l}\text { PI3K- } \\
\alpha\end{array}$ & $\begin{array}{l}\text { PI3K- } \\
\beta\end{array}$ & $\begin{array}{l}\text { PI3K- } \\
\delta\end{array}$ & $\begin{array}{l}\text { PI3K- } \\
\gamma\end{array}$ & AKT & mTOR & Trial ID & $\begin{array}{l}\text { Study } \\
\text { Phase }\end{array}$ \\
\hline $\begin{array}{l}\text { MENI6II + Trastuzumab } \pm \text { faslodex } \\
\text { AZD8I86 + docetaxel } \\
\text { Alpelisib + faslodex or letrozole } \\
\text { Alpelisib + nab-paclitaxel } \\
\text { Gedatolisib + palbociclib + faslodex } \\
\text { Gedatolisib + palbociclib/letrozole or palbociclib/faslodex } \\
\text { Ipatasertib + faslodex } \\
\text { Ipatasertib + aromatase inhibitor (Al) } \\
\text { Ipatasertib + faslodex + palbociclib } \\
\text { Ipatasertib + trastuzumab + pertuzumab + endocrine } \\
\text { therapy (if HR+) } \\
\text { Gedatolisib + PTK7-ADC } \\
\text { PDR00I (PDI monoclonal antibody) + everolimus, } \\
\text { LCLI6I or panobinostat } \\
\text { AZD5363 + paclitaxel } \\
\text { AZD20I4 + olaparib } \\
\text { AZD } 5363 \text { + olaparib } \\
\text { AZD20I4 + selumetinib (MEK inhibitor) } \\
\text { Taselisib +enzalutamide }\end{array}$ & $\begin{array}{l}x \\
x \\
x \\
x\end{array}$ & $\begin{array}{l}x \\
x\end{array}$ & $\begin{array}{l}x \\
x\end{array}$ & $\begin{array}{l}x \\
x\end{array}$ & $x$ & $x$ & $\begin{array}{l}\text { NCT03767335 } \\
\text { NCT032I } 8826 \\
\text { NCT03056755 } \\
\text { NCT042I } 6472 \\
\text { NCT02626507 } \\
\text { NCT02684032 } \\
\text { NCT0395989। } \\
\text { NCT0425356। } \\
\text { NCT0324333। } \\
\text { NCT02890069 } \\
\text { NCT02423603 } \\
\text { NCT02208375 } \\
\text { NCT02208375 } \\
\text { NCT02583542 } \\
\text { NCT024579I0 }\end{array}$ & $\begin{array}{l}\mathrm{I} \\
\mathrm{lb} \\
\mathrm{II} \\
\mathrm{lb} \\
\mathrm{lb} \\
\mathrm{lb} / \mathrm{ll} \\
\mathrm{lb} / \mathrm{II}\end{array}$ \\
\hline
\end{tabular}

study is evaluating the role of alpelisib in the treatment of HR+/HER2 negative PIK3CA mutated MBC. Initial results reported the cohort of patients with immediate prior treatment of CDK 4/6i $+\mathrm{AI}$ and went on to receive alpelisib + fulvestrant. At 6 months, the proportion of patients without disease progression was 50.4\% (95\% CI, 41.2-59.6). ${ }^{26}$ Additional cohorts are waiting to be reported, this includes patients with immediate prior treatment with CDK 4/6i+fulvestrant, systemic chemotherapy or endocrine therapy.

Alpelisib has also been studied in HER2 positive breast cancer. HER2 amplification and PIK3CA mutation can often both be seen simultaneously in breast cancer. This co-occurrence has been shown to have decreased response to HER2 directed therapies. ${ }^{27}$ Hanker and colleagues developed a mouse model of HER2+ and PIK3CA mutated breast cancers. It was found that dual HER2 amplification and PI3KCA mutation resulted in faster time to disease progression and conferred resistance to herceptin alone and in combination with pertuzumab and lapatinib. Interestingly, resistance was overcome with treatment with PI3K inhibitor. ${ }^{27}$ In a Phase I study by Jain and colleagues, patients with HER+ MBC who had progressed on taxane and trastuzumab therapy were treated with alpelisib in combination with T-DM1. ${ }^{28}$ Seventeen patients were enrolled and median number of prior therapies was three. In the fourteen patients that were evaluable for response, the overall response rate was $43 \%$. The most frequent toxicities included fatigue, rash, thrombocytopenia and anemia, hyperglycemia, elevated liver enzymes and gastrointestinal toxicities. ${ }^{28}$ These studies show promising results with alpelisib in the treatment of HER2 positive breast cancer but further studies will need to be conducted to confirm these findings.

\section{Conclusion}

The PI3K/mTOR pathway plays an important role in cell growth, proliferation and survival. Preclinical and clinical studies have demonstrated that inhibiting this pathway can be a key therapeutic strategy in the treatment of HR +/HER2 negative MBC. Alpelisib is the first PI3Ki approved for the treatment of $\mathrm{HR}+/ \mathrm{HER} 2$ negative $\mathrm{MBC}$ in patients who have a PIK3CA mutation and who have received prior endocrine therapy ${ }^{19}$ Further studies will need to be performed to evaluate the role of alpelisib in patients previously treated with CDK 4/6i and the role in other subtypes of breast cancer including HER2 positive disease. The development of alpelisib adds to the nonchemotherapy options for patients with HR+/HER2 negative MBC and is yet another demonstration of the shift in cancer care towards personalized medicine and targeted therapeutics. 


\section{Disclosure}

Dr Hyo Sook Han reports personal fees from Lilly, research funding to institution from Arvinas, Abbvie, BMS, Daiichi, G1 therapeutics, GSK, Horizon, Maker therapeutics, Novartis, Pfizer, Prescient, Seattle Genetics, Zymeworks, grants from Department of Defense, outside the submitted work. The authors report no other conflicts of interest in this work.

\section{References}

1. Society AC Breast cancer facts and figures 2019-2020. September 10, 2020; Available from: https://www.cancer.org/content/dam/can cer-org/research/cancer-facts-and-statistics/breast-cancer-facts-andfigures/breast-cancer-facts-and-figures-2019-2020.pdf. Accessed October 8, 2020.

2. Finn RS, Martin M, Rugo HS, et al. Palbociclib and letrozole in advanced breast cancer. $N$ Engl $J$ Med. 2016;375(20):1925. doi:10.1056/NEJMoa1607303

3. Goetz MP, Toi M, Campone M, et al. MONARCH 3: abemaciclib as initial therapy for advanced breast cancer. J Clin Oncol. 2017;35 (32):3638-3646. doi:10.1200/JCO.2017.75.6155

4. Hortobagyi GN, Stemmer SM, Burris HA, et al. Ribociclib as first-line therapy for HR-positive, advanced breast cancer. $N$ Engl $J$ Med. 2016;375(18):1738-1748. doi:10.1056/NEJMoa1609709

5. NCCN Clinical Practice Guidelines in Oncology (NCCN guidelines). Breast cancer. Version 3.2020. Available from: https://www.nccn.org/ professionals/physician_gls/pdf/breast.pdf. Accessed April 1, 2020.

6. Robertson JFR, Bondarenko IM, Trishkina E, et al. Fulvestrant $500 \mathrm{mg}$ versus anastrozole $1 \mathrm{mg}$ for hormone receptor-positive advanced breast cancer (FALCON): an international, randomised, double-blind, phase 3 trial. Lancet. 2016;388(10063):2997-3005. doi:10.1016/S0140-6736(16)32389-3

7. Baselga J, Campone M, Piccart M, et al. Everolimus in postmenopausal hormone-receptor-positive advanced breast cancer. $N$ Engl $J$ Med. 2012;366(6):520-529. doi:10.1056/NEJMoa1109653

8. Jones RH, Casbard A, Carucci M, et al. Fulvestrant plus capivasertib versus placebo after relapse or progression on an aromatase inhibitor in metastatic, oestrogen receptor-positive breast cancer (FAKTION): a multicentre, randomised, controlled, phase 2 trial. Lancet Oncol. 2020;21(3):345-357. doi:10.1016/S1470-2045(19)30817-4

9. Verret B, Cortes J, Bachelot T, Andre F, Arnedos M. Efficacy of PI3K inhibitors in advanced breast cancer. Ann Oncol. 2019;30(Suppl 10): x12-x20. doi:10.1093/annonc/mdz381

10. Fritsch C, Huang A, Chatenay-Rivauday C, et al. Characterization of the novel and specific PI3Kalpha inhibitor NVP-BYL719 and development of the patient stratification strategy for clinical trials. Mol Cancer Ther. 2014;13(5):1117-1129. doi:10.1158/1535-7163.MCT13-0865

11. LoRusso PM. Inhibition of the PI3K/AKT/mTOR pathway in solid tumors. $J$ Clin Oncol. 2016;34(31):3803-3815. doi:10.1200/ JCO.2014.59.0018

12. Cancer Genome Atlas Network. Comprehensive molecular portraits of human breast tumours. Nature. 2012;490(7418):61-70.

13. Stemke-Hale K, Gonzalez-Angulo AM, Lluch A, et al. An integrative genomic and proteomic analysis of PIK3CA, PTEN, and AKT mutations in breast cancer. Cancer Res. 2008;68(15):6084-6091. doi:10.1158/0008-5472.CAN-07-6854

14. Baselga J, Im S-A, Iwata H, et al. Buparlisib plus fulvestrant versus placebo plus fulvestrant in postmenopausal, hormone receptor-positive, HER2-negative, advanced breast cancer (BELLE-2): a randomised, double-blind, placebo-controlled, phase 3 trial. Lancet Oncol. 2017;18(7):904-916. doi:10.1016/S14702045(17)30376-5
15. Di Leo A, Johnston S, Lee KS, et al. Buparlisib plus fulvestrant in postmenopausal women with hormone-receptor-positive, HER2-negative, advanced breast cancer progressing on or after mTOR inhibition (BELLE-3): a randomised, double-blind, placebo-controlled, phase 3 trial. Lancet Oncol. 2018;19(1):87-100. doi:10.1016/S1470-2045(17)30688-5

16. Vuylsteke P, Huizing M, Petrakova K, et al. Pictilisib PI3Kinase inhibitor (a phosphatidylinositol 3-kinase [PI3K] inhibitor) plus paclitaxel for the treatment of hormone receptor-positive, HER2-negative, locally recurrent, or metastatic breast cancer: interim analysis of the multicentre, placebo-controlled, phase II randomised PEGGY study. Ann Oncol. 2016;27(11):2059-2066.

17. Baselga J, Dent SF, Cortés J, et al. Phase III study of taselisib (GDC-0032) + fulvestrant (FULV) v FULV in patients (pts) with estrogen receptor (ER)-positive, PIK3CA -mutant (MUT), locally advanced or metastatic breast cancer (MBC): primary analysis from SANDPIPER. $\quad J$ Clin Oncol. 2018;36(18 suppl):LBA1006LBA1006. doi:10.1200/JCO.2018.36.18_suppl.LBA1006

18. Juric D, Rodon J, Tabernero J, et al. Phosphatidylinositol 3-kinase $\alpha-$ selective inhibition with alpelisib (BYL719) in PIK3CA -altered solid tumors: results from the first-in-human study. J Clin Oncol. 2018;36 (13):1291-1299. doi:10.1200/JCO.2017.72.7107

19. FDA highlights of prescribing information: alpelisib. Version 5.2019. Available from: https://www.accessdata.fda.gov/drugsatfda_docs/ label/2019/212526s000lbl.pdf. Accessed April 3, 2020

20. Juric D, Janku F, Rodón J, et al. Alpelisib plus fulvestrant in PIK3CA -altered and PIK3CA -wild-type estrogen receptor-positive advanced breast cancer. JAMA Oncol. 2019;5(2):e184475. doi:10.1001/ jamaoncol.2018.4475

21. Andre F, Ciruelos E, Rubovszky G, et al. Alpelisib for PIK3CA mutated, hormone receptor-positive advanced breast cancer. $N$ Engl J Med. 2019;380(20):1929-1940. doi:10.1056/NEJMoa1813904

22. Brandão M, Caparica R, Eiger D, et al. Biomarkers of response and resistance to $\mathrm{PI} 3 \mathrm{~K}$ inhibitors in estrogen receptor-positive breast cancer patients and combination therapies involving PI3K inhibitors. Ann Oncol. 2019;30(Suppl_10):x27-x42. doi:10.1093/ annonc/mdz280

23. Razavi P, Dickler MN, Shah PD, et al. Alterations in PTEN and ESR1 promote clinical resistance to alpelisib plus aromatase inhibitors. Nat Cancer. 2020;1(4):382-393. doi:10.1038/s43018020-0047-1

24. Vasan N, Razavi P, Johnson JL, et al. Double PIK3CA mutations in cis increase oncogenicity and sensitivity to $\mathrm{PI} 3 \mathrm{~K} \alpha$ inhibitors. Science. 2019;366(6466):714-723.

25. Martínez-Sáez O, Chic N, Pascual T, et al. Frequency and spectrum of PIK3CA somatic mutations in breast cancer. Breast Cancer Res. 2020;22(1):45. doi:10.1186/s13058-020-01284-9

26. Rugo HS, Lerebours F, Ciruelos E, et al. Alpelisib (ALP) + fulvestrant (FUL) in patients (pts) with PIK3CA-mutated (mut) hormone receptor-positive $(\mathrm{HR}+)$, human epidermal growth factor receptor 2-negative (HER2-) advanced breast cancer (ABC) previously treated with cyclin-dependent kinase 4/6 inhibitor (CDKi) + aromatase inhibitor (AI): bYLieve study results. J Clin Oncol. 2020;38 (15_suppl): 1006 .

27. Hanker AB, Pfefferle AD, Balko JM, et al. Mutant PIK3CA accelerates HER2-driven transgenic mammary tumors and induces resistance to combinations of anti-HER2 therapies. Proc Natl Acad Sci U S A. 2013;110(35):14372-14377. doi:10.1073/pnas.1303204110

28. Jain S, Shah AN, Santa-Maria CA, et al. Phase I study of alpelisib (BYL-719) and trastuzumab emtansine (T-DM1) in HER2-positive metastatic breast cancer (MBC) after trastuzumab and taxane therapy. Breast Cancer Res Treat. 2018;171(2):371-381. doi:10.1007/s10549018-4792-0

29. Costa RLB, Han HS, Gradishar WJ. Targeting the PI3K/AKT/mTOR pathway in triple negative breast cancer: a review. Breast Cancer Res Treat. 2018;169(3):397-406. doi:10.1007/s10549-018-4697-y 


\section{Publish your work in this journal}

Breast Cancer - Targets and Therapy is an international, peer-reviewed open access journal focusing on breast cancer research, identification of therapeutic targets and the optimal use of preventative and integrated treatment interventions to achieve improved outcomes, enhanced survival and quality of life for the cancer patient.
The manuscript management system is completely online and includes a very quick and fair peer-review system, which is all easy to use. Visit http://www.dovepress.com/testimonials.php to read real quotes from published authors. 